(C) 2020 Universidad Nacional Autónoma de México, Facultad de Estudios Superiores Zaragoza.

Este es un artículo Open Access bajo la licencia CC BY-NC-ND (http://creativecommons.org/licenses/by-nc-nd/4.0/).

TIP Revista Especializada en Ciencias Químico-Biológicas, 23: 1-12, 2020.

DOI: 10.22201 /fesz.23958723e.2020.0.224

\title{
Hidrogeles de colágeno acoplados con hidroxiapatita para aplicaciones en ingeniería tisular
}

\author{
Rosalina Lara-Rico, Jesús Alejandro Claudio-Rizo, \\ Elia Martha Múzquiz-Ramos y Claudia Magdalena Lopez-Badillo* \\ Facultad de Ciencias Químicas, Universidad Autónoma de Coahuila, Blvd. Venustiano Carranza e \\ Ing. José Cárdenas Valdés s/n, Saltillo 25280, Coahuila, México. E-mail: *cllopezb@uadec.edu.mx
}

\begin{abstract}
RESUMEN
Los hidrogeles basados en colágeno son redes tridimensionales (3D) con la capacidad de absorber agua y una alta biocompatibilidad para utilizarlos en la reparación de tejidos dañados. Estos materiales presentan pobres propiedades mecánicas y velocidades de degradación rápidas, limitando su aplicación a estrategias de ingeniería tisular y biomedicina; por ésto, la incorporación de fases inorgánicas en la matriz 3D del colágeno como la hidroxiapatita ha contribuido en la mejora de sus propiedades, incrementado la eficiencia de los hidrogeles híbridos obtenidos. Este trabajo, presenta las contribuciones más relevantes relacionadas con los sistemas de hidrogeles basados en colágeno y partículas de hidroxiapatita dispersas dentro de la matriz colagénica, lo que evidencia que la combinación de los materiales no altera la biocompatibilidad y biodegradabilidad típicas del colágeno, permitiendo la adhesión, proliferación, crecimiento celular y control del metabolismo de las células implicadas en los procesos de una reparación ósea, presentando a los hidrogeles como una estrategia para su uso potencial en la ingeniería tisular.
\end{abstract}

Palabras clave: hidroxiapatita, ingeniería tisular, hidrogel, colágeno.

\section{Collagen hydrogels coupled with hydroxyapatite for tissue engineering applications}

\begin{abstract}
Collagen based hydrogels are three dimensional (3D) networks with the capacity to absorb water and high biocompatibility for use in repairing damaged tissue. These materials have poor mechanical properties and rapid degradation rates, limiting their application to tissue engineering and biomedical strategies; for this reason, the incorporation of inorganic phases in the 3D matrix of collagen such as hydroxyapatite has contributed to improving its properties, increasing the efficiency of the hybrid hydrogels obtained. This work presents the most relevant contributions related to collagen-based hydrogel systems and hydroxyapatite particles dispersed within the collagen matrix, which shows that the combination of materials does not alter the typical biocompatibility and biodegradability of collagen, allowing adhesion, proliferation, cell growth and control of the metabolism of the cells involved in the processes of bone repair, presenting hydrogels as a strategy for their potential use in tissue engineering.
\end{abstract}

Keywords: hydroxyapatite, tissue engineering, hydrogel, collagen. 


\section{INTRODUCCIÓN}

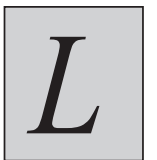

as enfermedades, lesiones y traumatismos pueden provocar daños y degeneración no reversible en los diferentes tejidos del cuerpo humano, lo que requiere de diversos tratamientos médicos para su reparación, reemplazo o regeneración.

Los tratamientos usualmente empleados consisten en dos tipos de procedimientos: uno de ellos es el autoinjerto y el otro es un aloinjerto (o trasplante).

El tipo de injerto más común es un autoinjerto, en este tipo de procedimiento se toma un hueso del cuerpo del paciente y es reimplantado, sin embargo, hay una cantidad limitada de hueso que puede ser utilizado, además de que se requiere de una cirugía invasiva adicional. La recolección de autoinjertos es costosa, dolorosa y se asocia con la morbilidad del sitio donante, debido a posibles infecciones y hematomas.

Otro tratamiento alternativo es el uso de un aloinjerto mediante el cual se extrae hueso del donante, con alto riesgo de transmisión de enfermedades infecciosas, además de la escasez de donantes compatibles. Los aloinjertos o trasplantes también presentan serias limitaciones debido a los problemas para acceder a suficiente tejido, al riesgo de rechazo por parte del sistema inmunitario del paciente y a la posibilidad de introducir alguna infección o enfermedad del donante al paciente, por ello se ha investigado acerca de la ingeniería de tejidos óseos como una alternativa a los tratamientos antes mencionados (Al-Munajjed et al., 2009; Ambekar \& Kandasubramanian, 2019; O’Brien, 2011; Roberts \& Rosenbaum, 2012).

La ingeniería tisular es un área multidisciplinaria que aplica los principios de la ingeniería y de las ciencias de la salud para el desarrollo de estructuras biológicas, con el fin de generar tejidos que permitan restaurar, mantener o mejorar la función del tejido u órgano afectado. Es una ciencia en continuo desarrollo que presenta innumerables posibilidades terapéuticas; algunos productos logrados con esta tecnología han sido aprobados por la FDA(Food and Drug Administration) y muchos se encuentran en fases avanzadas de ensayos clínicos para su posterior aplicación (Orive, Hernández, Garcón, Igartúa \& Pedráz M., 2003).

Actualmente, es posible fabricar implantes para cualquier parte del cuerpo humano, ya existen diferentes materiales que pueden ser empleados dependiendo del tejido que se va a reemplazar. Por ejemplo, el desarrollo de nuevos biomateriales como los andamios para la ingeniería tisular que ayudan a mantener la función durante la regeneración y como plantilla en las interacciones celulares necesarias para la reparación del tejido (Cosgriff-Hernandez \& Mikos, 2008).

La ingeniería tisular requiere de la implantación de materiales porosos que proporcionen el soporte necesario para aprovechar la progresión natural del crecimiento óseo aposicional y la proliferación de las células en el tejido óseo (Lickorish, Ramshaw, Werkmeister, Glattauer \& Howlett, 2004).

Recientemente, el uso de biomateriales en estado hidrogel representa una estrategia innovadora para lograr la formación de un nuevo tejido óseo, debido a las propiedades que estos materiales presentan, como hinchamiento y difusión de metabolitos de interés, capacidad de adaptarse al sitio de la lesión sin necesidad de emplear técnicas quirúrgicas complicadas gelificando en el lecho de la lesión, capacidad de carga con biomoléculas para mejorar la respuesta biológica de los procesos de reparación tisular y degradación adaptada permitiendo la generación del nuevo tejido sin la formación de defectos que pongan en riesgo su función original (Bahram, Mohseni \& Moghater, 2015; Chai, Jiao \&Yu, 2017; Lammi, Piltti, Prittinen \& Qu, 2018).

Los hidrogeles basados en colágeno, han mostrado que su aplicación es una estrategia potencial en ingeniería tisular, por su alta biocompatibilidad y capacidad para modular el metabolismo de las células implicadas en los procesos de reparación de tejido óseo (Bai et al., 2018) debido a la estructura fibrilar del colágeno que puede permitir la correcta dispersión de la hidroxiapatita (HAp), que es una fase inorgánica de composición similar a la de los huesos humanos, con buena biocompatibilidad, osteconductividad, y ha sido usada en muchas aplicaciones biomédicas (Bharti, Singh, Meena \& Goyal, 2016; Lin \& Chang, 2015). En este artículo se abordarán inicialmente los aspectos generales del tejido óseo, los biomateriales empleados para ingeniería tisular y los hidrogeles enfocados a los de colágeno acoplados con hidroxiapatita, ya que este tipo de compósitos representan una estrategia clave para la correcta reparación de los tejidos óseos dañados y por ser actualmente un área de investigación prometedora que busca la adaptación de sus propiedades para aplicaciones exitosas en ingeniería tisular.

\section{Hueso Biológico \\ Composición química}

El hueso está compuesto principalmente de: colágeno (20\% en peso), fosfato de calcio ( $69 \%$ en peso) y agua $(9 \%$ en peso), además de otros materiales orgánicos como proteínas, polisacáridos y lípidos presentes en pequeñas cantidades (Suchanek \& Yoshimura, 1998; Szcześ, Hołysz \& Chibowski, 2017).

El hueso natural es considerado un híbrido de tejido inorgánicoorgánico compuesto de cristales de HAp (hidroxiapatita) y nanofibras de colágeno ensamblados en una estructura altamente porosa. Se compone principalmente de una fase orgánica (proteínas) que contiene colágeno como matriz y una fase inorgánica (minerales) compuesta de hidroxiapatita principalmente (Tabla I) (Hutmacher, Schantz, Lam, Tan 
\& Lim, 2007; Murugan \& Ramakrishna, 2005; Peng, Yu \& Wei, 2011).

Tabla I. Composición general del hueso (Fillingham \& Jacobs, 2016; Giannoudis, Dinopoulos \& Tsiridis, 2005; Murugan \& Ramakrishna, 2005; Oftadeh, Perez-Viloria, Villa-Camacho, Vaziri \& Nazarian, 2015).

\begin{tabular}{|l|l|}
\hline \multicolumn{2}{|c|}{ Composición química del hueso } \\
\hline \multicolumn{1}{|c|}{ Parte orgánica } & Parte inorgánica (fase mineral) \\
\hline Colágeno & Carbonato de calcio \\
Polisacáridos, lípidos, & Fosfato de Calcio \\
proteínas & Hidroxiapatita $(\mathrm{HAp})$ \\
Células Óseas & $\mathrm{Na}^{+}, \mathrm{Mg}^{2+}, \mathrm{Cl}^{-}, \mathrm{Fe}^{2+}, \mathrm{F}^{-}, \mathrm{Cu}^{2+}, \mathrm{Sr}^{2+}$, \\
- Osteoblastos & $\mathrm{Zn}^{2+}$ \\
- Osteocitos & \\
- Osteoclastos & \\
Tejido conectivo & \\
\hline \multicolumn{2}{|l}{ Agua } \\
\hline
\end{tabular}

\section{Función}

El hueso es un tejido dinámico, altamente vascularizado y con una capacidad única para sanar y regenerarse sin dejar cicatrices, estas propiedades junto con su capacidad para movilizar rápidamente las reservas de minerales según la demanda metabólica lo convierten en el mejor material inteligente. Su principal función es proporcionar estructura y apoyo para el cuerpo humano, además también sirve como depósito de minerales, soporta la contracción muscular que se produce con el movimiento, resiste la carga y protege los órganos internos, por ello cuando se sufre de una lesión o enfermedad se puede alterar drásticamente el equilibrio corporal y la calidad de vida de la persona (Salgado, Coutinho \& Reis, 2004; Sommerfeldt \& Rubin, 2001).

\section{Desgaste natural o patológico y su reparación}

La importancia del hueso se refleja en el gran impacto económico y clínico de los tratamientos de defectos óseos como resultado de enfermedades como osteogénesis imperfecta, osteoartritis, osteomielitis y osteoporosis (Porter, Ruckh \& Popat, 2009). Además, el envejecimiento de la población, así como la obesidad y mala actividad física ha llevado a un incremento de la aparición de trastornos óseos que incluyen fracturas óseas, lumbalgia, escoliosis, osteoporosis, infección ósea, tumores y enfermedades reumáticas. De hecho, más de 20 millones de personas al año en todo el mundo se ven afectadas por una pérdida de tejido óseo causada por un trauma o enfermedad, lo que representa un grave problema que requiere estrategias de prevención y/o control (De Witte, Fratila-Apachitei, Zadpoor \& Peppas, 2018).

El tejido óseo presenta una excelente capacidad de regeneración y el hueso puede ser reparado en respuesta a un trauma o lesión, sin embargo, si la lesión es muy grave, la regeneración del hueso se ve deteriorada y puede llegar a requerir de una intervención quirúrgica (Dimitriou, Jones, McGonagle \& Giannoudis, 2011). Aunque se han realizado importantes avances en el área de la medicina regenerativa ósea, las terapias actuales como los injertos óseos aún presentan diversas limitaciones y la mayoría de las lesiones graves relacionadas con el hueso no son tratadas adecuadamente (Amini, Laurencin \& Nukavarapu, 2012).

\section{Biomateriales Generalidades}

Se han realizado diversas investigaciones por parte de científicos en las áreas de biomedicina y de ingeniería de materiales para desarrollar un material basado en componentes naturales $\mathrm{y} / \mathrm{o}$ sintéticos, capaz de ser implantado en el cuerpo, lo que se conoce como un biomaterial.

Un "biomaterial" está definido por Park, como cualquier material que reemplace la función de los tejidos u órganos, además de ser una sustancia farmacológicamente inerte diseñada para implantarse o incorporarse a un sistema vivo (Joon, 1984; Laurencin, Ambrosio, Borden \& Cooper, 1999).

Un material empleado en ingeniería tisular debe de poseer algunas propiedades básicas y cumplir con los objetivos de la regeneración ósea artificial, permitir o mejorar la viabilidad celular, favorecer la unión, la proliferación y la ubicación espacial de las células, permitir la diferenciación osteogénica, la vascularización, la integración con el huésped y cuando sea necesario soportar la carga mecánica adecuada. Además, debe de ser no tóxico, biocompatible, biodegradable, bioactivo, osteoconductor, con forma y tamaño ajustables (Martin-Piedra \& Martin-Piedra, 2019; Turnbull et al., 2018; Yahia, Khalil \& El-Sherbiny, 2019).

\section{Biomateriales empleados en ingeniería tisular}

Generalmente, los biomateriales son los más empleados y se dividen en cerámicos, metales, materiales compuestos (compósitos) y polímeros, cada grupo exhibe algunas ventajas y desventajas, por ejemplo, los cerámicos son materiales biocompatibles que se pueden obtener con propiedades bioestables, bioactivas o bioabsorbibles, pero sus principales inconvenientes son su dureza y fragilidad; los metales tienen problemas de corrosión y toxicidad pero su comportamiento mecánico es óptimo; los polímeros presentan muchas posibilidades dependiendo de su composición química y estructura (grado de biodegradabilidad, flexibilidad) pero muy pocos han mostrado buenas propiedades bioactivas para asegurar la osteointegración del implante, por ello es común utilizar los tres tipos de materiales en el mismo implante con el fin de obtener un mejor resultado, generando así diversos compósitos. Por ejemplo, es el caso de una prótesis total de cadera que presenta un haz de metal, parcialmente recubierto con una cerámica bioactiva, así como de materiales poliméricos como el polietileno (Vallet-Regí, 2010). 
En ingeniería tisular, los hidrogeles y elastómeros termoplásticos se encuentran entre las categorías de andamios óseos comúnmente empleados, también las cerámicas bioactivas como los fosfatos de calcio pueden ser utilizados. Los compuestos de fosfato de calcio incluyendo la hidroxiapatita, fosfato $\alpha$-tricálcico $(\alpha$-TCP) y fosfato $\beta$-tricálcico $(\beta$-TCP) son más utilizados por brindar una buena resistencia mecánica (Zhao, Ma, Gao \& Shen, 2008).

Existen diversos materiales empleados en ingeniería de tejidos, estos se pueden dividir en materiales naturales y sintéticos. En la Tabla II se mencionan algunos ejemplos.

\section{Hidrogeles}

Un hidrogel es una red 3D constituida por polímeros altamente reticulados mediante interacciones fisicoquímicas con la capacidad de absorber grandes cantidades de agua, capacidad que surge de los grupos funcionales hidrofílicos unidos a la estructura polimérica, mientras que su resistencia a la disolución se debe a los enlaces de entrecruzamiento formados entre las cadenas de la red.

Los hidrogeles pueden ser naturales o sintéticos, estos últimos presentan una larga vida útil, alta resistencia y estructuras bien definidas, que pueden modificarse para evaluar la degradabilidad y funcionalidad del gel (Ahmed, 2015).
Los sistemas híbridos de los hidrogeles son una estrategia clave para la correcta reparación de tejidos óseos dañados. Sin embargo, a la fecha el desarrollo de compósitos en estado hidrogel basado en el colágeno es un área de investigación prometedora que busca la adaptación de las propiedades de estos biomateriales inteligentes para aplicaciones exitosas en ingeniería tisular. Estas estrategias involucran el uso de polisacáridos, como el alginato o quitosano por mencionar algunos, que son de gran interés para su posible uso en el desarrollo y diseño de andamios con propiedades mejoradas y aplicación en biomedicina (Kaviani, Zebarjad, Javadpour, Ayatollahi \& Bazargan-Lari, 2019), demostrando control en la respuesta biológica y modificación de los fenotipos celulares que permiten la reparación de los tejidos dañados.

Los hidrogeles suelen ser empleados como matrices en ingeniería de tejidos, en medicina regenerativa y utilizarse para imitar la topografía de la matriz extracelular con los agentes bioactivos necesarios que promueven la regeneración del tejido óseo.

Los hidrogeles se caracterizan por presentar contenido muy similar al tejido óseo, estabilidad de la estructura y la encapsulación celular homogénea conduce a una mejora en la biocompatibilidad minimizando el daño al tejido adyacente; son químicamente biocompatibles y biodegradables, altamente porosos, mínimamente antigénicos y se pueden combinar

Tabla II. Ejemplos de polímeros naturales y sintéticos utilizados para aplicaciones en ingeniería de tejidos.

\begin{tabular}{|c|c|c|c|}
\hline Material & Origen & Características & Referencias \\
\hline Colágeno & Natural & $\begin{array}{l}\text { - Favorece la adhesión celular. } \\
\text { - Puede ser empleado como andamio. } \\
\text { - Quimiotáctico. }\end{array}$ & $\begin{array}{l}\text { (Sachlos, Reis, Ainsley, Derby } \\
\text { \& Czernuszka, 2003) }\end{array}$ \\
\hline Fibrina & Natural & $\begin{array}{l}\text { - Promueve la migración celular y la vascularización. } \\
\text { - Promueve la osteoconducción. } \\
\text { - Por lo general, se usa como portador celular para la siembra } \\
\text { celular en andamios. }\end{array}$ & $\begin{array}{l}\text { (Froelich et al., 2010, Salgado } \\
\text { et al., 2004) }\end{array}$ \\
\hline Quitosano & Natural & $\begin{array}{l}\text { - Posee propiedades antibacterianas y antifúngicas. } \\
\text { - Actividad inmunológica. } \\
\text { - Buena biocompatibilidad y biodegradabilidad. }\end{array}$ & $\begin{array}{l}\text { (Tolaimate, Desbrieres, Rhazi } \\
\text { \& Alagui, 2003; Zivanovic, } \\
\text { Davis \& Golden, 2015) }\end{array}$ \\
\hline Almidón & Natural & $\begin{array}{l}\text { - Biopolímero biodegradable. } \\
\text { - No tóxico. } \\
\text { - Biocompatible y de bajo costo. } \\
\text { - Es empleado como biomaterial en administración de fármacos. }\end{array}$ & $\begin{array}{l}\text { (George, Shah \& Shrivastav, } \\
\text { 2019; Imre \& Pukánszky, 2013; } \\
\text { Xu, Tan, Chen, Li \& Xie, 2019, } \\
\text { Khan \& Ahmad, 2013) }\end{array}$ \\
\hline $\begin{array}{c}\text { Poli (ácido } \\
\gamma \text {-glutámico) }\end{array}$ & Natural & $\begin{array}{l}\text { - Buena biodegradabilidad y biocompatibilidad. } \\
\text { - No tóxico. } \\
\text { - Muestra actividad antinflamatoria. }\end{array}$ & $\begin{array}{l}\text { (Lee et al., 2018; Shi et al., } \\
\text { 2015) }\end{array}$ \\
\hline $\begin{array}{c}\text { Poli } \\
\text { (e-caprolactona) }\end{array}$ & Sintético & $\begin{array}{l}\text { - Miscibilidad con otros polímeros. } \\
\text { - Buena biodegradabilidad. } \\
\text { - Degradado por hidrólisis. }\end{array}$ & $\begin{array}{l}\text { (Gea et al., 2018; Labet \& } \\
\text { Thielemans, 2009) }\end{array}$ \\
\hline
\end{tabular}


fácilmente con otros materiales (De Witte, Fratila-Apachitei, Zadpoor \& Peppas, 2018). Pueden ser procesados en condiciones suaves, tener propiedades mecánicas adaptadas y aplicarse de forma mínimamente invasiva (Tan \& Marra, 2010).

Existe gran variedad de materiales sintéticos y derivados naturales que pueden ser empleados para formar hidrogeles y ser utilizados como andamios en ingeniería de tejidos. Algunos ejemplos de materiales sintéticos son el óxido de polietileno (PEO), polialcohol vinílico (PVA), ácido poliacrílico (PAA) y como materiales naturales la agarosa, alginato, quitosano, colágeno, fibrina, gelatina y ácido hialurónico (Drury \& Mooney, 2003).

\section{Propiedades mecánicas de los hidrogeles}

El desempeño mecánico de un hidrogel puede ser estudiado si se conocen sus módulos de almacenamiento ( $\left.G^{\prime}\right)$, ya que indica la capacidad de la matriz para soportar cargas o comportarse como un sólido, y su módulo viscoso o de pérdida (G") se relaciona con la capacidad de fluir del hidrogel relacionado con el comportamiento de un líquido. Un hidrogel siempre estará caracterizado por un G' mayor que un G" (Chen et al., 2017). Estrategias que permitan controlar el G' de sistemas en estado hidrogel son necesarias para la generación de hidrogeles con potencial aplicación en ingeniería tisular. Se ha reportado que los hidrogeles de matriz extracelular (ECM) presentan un módulo de almacenamiento de 600-700 Pa para tejidos cartilaginosos y tendones (Claudio-Rizo et al., 2017). El desempeño adecuado de un hidrogel en estrategias de ingeniería tisular depende de controlar las propiedades mecánicas del material, incluyendo la elasticidad, compresibilidad, comportamiento viscoelástico, resistencia a la tracción y falla a la tensión, lo cual puede ser regulado controlando el G', ya que la formación de un nuevo tejido requiere que la mecánica del hidrogel sea adaptable evitando la generación de tejidos defectuosos, así como también asegurar que la vida útil del hidrogel es la adecuada durante el proceso de regeneración (Anseth, Bowman \& BrannonPeppas, 1996).

\section{Hidroxiapatita}

Diversos materiales cerámicos como la alúmina, circonia, fosfatos de calcio e hidroxiapatita han sido empleados en el área de la medicina desde hace mucho tiempo, debido a que presentan buena biocompatibilidad, baja densidad, estabilidad química, alta resistencia al desgaste y en el caso de los fosfatos de calcio y la hidroxiapatita, además de lo anterior, su composición es similar a la fase mineral del hueso. Los fosfatos de calcio tienen efectos osteconductivos y pueden, en ciertas condiciones, unirse al hueso. Se ha reportado en ensayos in vivo e in vitro que los fosfatos de calcio ya sea en forma compacta, porosos, como recubrimientos o en polvo, soportan la unión, diferenciación y proliferación de células como los osteoblastos y las células mesenquimatosas. Uno de los fosfatos de calcio más eficientes es la HAp debido a su estabilidad ante cambios de $\mathrm{pH}$, temperatura y composición del fluido fisiológico (Kalita, Bhardwaj \& Bhatt, 2007; Lin \& Chang, 2015; Rincón, Rodríguez, Londoño \& Echavarría, 2007).

La HAp puede ser obtenida de manera sintética, empleando métodos como: el hidrotermal (Shuk et al., 2001), la mecanosíntesis (Nasiri-Tabrizi, Fahami \& EbrahimiKahrizsangi, 2013), el sol-gel (Kuriakose et al., 2004) o el de precipitación (Ramli, Adnan, Bakar \& Masudi, 2011), permitiendo seleccionar la relación de $\mathrm{Ca} / \mathrm{P}$, el tamaño del grano, la cristalinidad, porosidad y pureza, entre otras características (Rincón, Rodríguez, Londoño \& Echavarría, 2007).

LaHAp es el mayor componente inorgánico (mineral) del hueso y se considera el material más utilizado en implantes óseos, debido a su composición y características biológicas similares con el hueso natural. La HAp se ha empleado en varias aplicaciones biomédicas por su excelente bioactividad, promoción de la función celular y osteoconductividad. Además, varios autores señalan que la HAp puede unirse directamente a los tejidos y promover su crecimiento. Su principal problema es su baja resistencia mecánica, por lo que cada vez es menos utilizada en implantes que requieren permanecer largo tiempo y soportar alta carga (Sadat-Shojai, Khorasani, Dinpanah-Khoshdargi \& Jamshidi, 2013; Sequeda, Díaz, Gutiérrez, Perdomo \& Gómez, 2012; Zhou \& Lee, 2011).

LaHAp ha sido utilizada ampliamente en cirugía reconstructiva ortopédica y dental, tanto como relleno masivo de huecos óseos o como recubrimiento de superficies, ya que promueve la adhesión entre prótesis y el hueso (Silva, Pinheiro, Miranda, Góes \& Sombra, 2003).

\section{Colágeno}

Es el componente principal de la piel y los huesos (tipo I), también se puede encontrar en cartílagos (tipo II) y en los vasos sanguíneos (tipo III), representa el 30\% de la masa de los vertebrados además de contar con excelentes propiedades biocompatibles, osteconductoras y osteointegrativas. Generalmente se utiliza como expansor de injerto óseo, pero también puede ser empleado como andamio para deposición mineral. El colágeno se degrada fácilmente y es reabsorbido por el cuerpo permitiendo una buena adhesión celular, sin embargo, sus propiedades mecánicas son relativamente bajas en comparación con el hueso (Carlson et al., 2004; Sherman, Yang \& Meyers, 2015; Wahl \& Czernuszka, 2006; Wang \& Yeung, 2017).

Los biomateriales a base de colágeno son de suma importancia para la ingeniería de tejidos y en medicina regenerativa debido a su excelente biocompatibilidad y baja inmunogenicidad.

Los andamios de colágeno pueden mejorar la migración, proliferación y diferenciación celular. Actualmente se sabe que 
existen 29 tipos diferentes de colágeno, sin embargo, el colágeno tipo I es el más empleado para aplicaciones en ingeniería de tejidos óseos (Jansen et al., 2018; Parenteau-Bareil, Gauvin \& Berthod, 2010).

Para la mayoría de los tejidos conectivos blandos y duros (huesos, cartílago, tendón, córnea, vasos sanguíneos y piel) las fibrillas de colágeno y sus redes funcionan como una matriz extracelular (EMC), la cual brinda movilidad y flexibilidad definiendo comportamientos celulares y función tisular (Cen, Liu, Cui, Zhang \& Cao, 2008).

Debido a su estructura, el colágeno promueve la deposición de minerales y favorece la adhesión celular (Parikh, 2002). La función de las fibras de colágeno es proporcionar resistencia en tensión y resistencia a la flexión, mientras que los cristales de apatita incrustados entre las nano fibras es resistir la compresión, en las redes de colágeno ocurre la regeneración fisiológica (Gardin et al., 2012).

\section{Hidrogeles de colágeno acoplados con hidroxiapatita}

Los materiales a base de colágeno han sido empleados en ingeniería tisular debido a la baja reacción inmunológica, a su biocompatibilidad y podrían proporcionar apoyo para la proliferación celular. Los hidrogeles de colágeno presentan algunos inconvenientes para su aplicación debido a la rápida degradación hidrolítica y enzimática, y a la baja resistencia mecánica que presentan, por ello la incorporación de materiales inorgánicos en hidrogeles poliméricos puede ser una opción adecuada para superar estos problemas y mejorar el desempeño biológico (Figura 1). Los materiales cerámicos a base de fosfato de calcio son un tipo de materiales inorgánicos utilizados para la regeneración ósea. Entre los materiales con fosfato de calcio, la hidroxiapatita ha sido utilizada ampliamente en los hidrogeles a base de colágeno para aumentar su rigidez (Takallu, Mirzaei, Azadi, Karimizade \& Tavakol, 2019). La mejoría en las propiedades mecánicas y velocidad de degradación está relacionada con la formación de puentes de hidrógeno entre los grupos $\mathrm{P}-\mathrm{O}$ de la hidroxiapatita con los grupos $-\mathrm{NH}$ y $\mathrm{COOH}$ del colágeno (Stock, 2015). Sin embargo, un exceso de fase inorgánica puede impedir que la red fibrilar de colágeno polimerice evitando la formación del hidrogel; siendo limitada la concentración de hidroxiapatita dentro de la matriz3D colagénica.

Las propiedades mecánicas de los hidrogeles de colágeno dependen de su estructura fibrilary de las asociaciones que tengan con la diversidad de moléculas tanto de la matriz extracelular como de las exógenas, determinando su capacidad para absorber agua e hinchamiento (AbouNeel, Cheema, Knowles, Brown \& Nazhat, 2006).

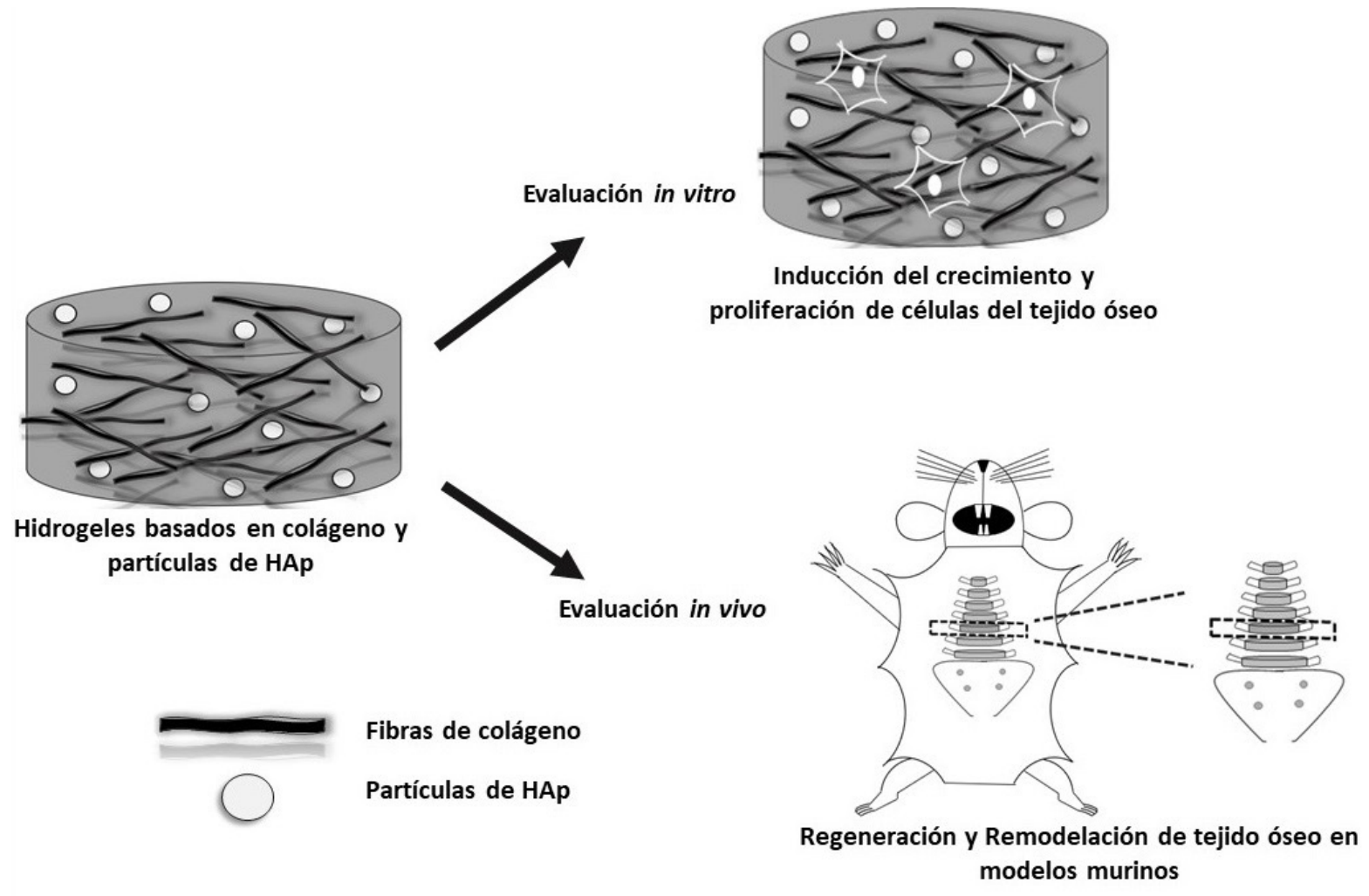

Figura 1. Esquema de la aplicación de los hidrogeles con HAp en estrategias de regeneración tisular ósea. Elaboración personal. 
En tejidos óseos la formación de fibras de colágeno debe ser direccionada por fases inorgánicas como la HAp. La nanoestructura, cristalinidad y morfología de la HAp contribuyen a la generación de hidrogeles basados en fibras de colágeno que permitan modular su comportamiento para aplicaciones en ingeniería tisular (Wang et al., 2013).

Los biomateriales de colágeno con hidroxiapatita han demostrado tener un efecto de curación ósea acelerada y una mejor osteointegración debido al uso de la mezcla de ambos materiales. En los compuestos de HAp/colágeno este último tiene la función de regular la distribución de las partículas de la parte cerámica evitando la aglomeración como se observa en la Figura 2 (Zhang et al., 2004).

Como se mencionó anteriormente, los andamios de colágeno puro presentan una resistencia mecánica sumamente baja, por ello mediante la adición de HAp se puede lograr un aumento en el módulo elástico ( 0.66-0.97 MPa), así como en la superficie de los andamios, lo que conducirá a una mayor adhesión celular (Li et al., 2013; Zhang, Wu, Chen \& Lin, 2018).

Se ha reportado que los andamios de nanocompuestos de colágeno con hidroxiapatita exhiben una distribución uniforme de las partículas inorgánicas en el hidrogel de colágeno, ayudando a que las células se adhieran y crezcan en los andamios porosos híbridos, logrando una buena interacción ente la matriz polimérica y la fase inorgánica. El tamaño nanométrico de la fase inorgánica es importante, ya que puede ser internalizada en las células involucradas en los procesos de curación del tejido óseo promoviendo una regeneración acelerada (Chen, Hu, Ran, Shen \&Tong, 2016).

En la Tabla III, se muestran algunas de las investigaciones más relevantes de hidrogeles de colágeno e hidroxiapatita en estrategias de ingeniería tisular.

En los hidrogeles de colágeno mediante la incorporación de diferentes materiales, principalmente inorgánicos (cerámicos), se puede lograr un aumento en las propiedades tanto mecánicas como biológicas, según se observa en la Tabla III, ya que favorecen el incremento de las propiedades mecánicas de los hidrogeles ante la presencia de HAp; se ha reportado un aumento en el desempeño mecánico en comparación con el colágeno puro; el andamio compósito resiste una mayor tensión y no muestra algún tipo de fractura, además de presentar una distribución homogénea. Por otra parte, la presencia de materiales naturales como el ácido hialurónico, quitosano y alginato, también favorecen las propiedades mecánicas y biológicas principalmente de adhesión, proliferación, crecimiento celular

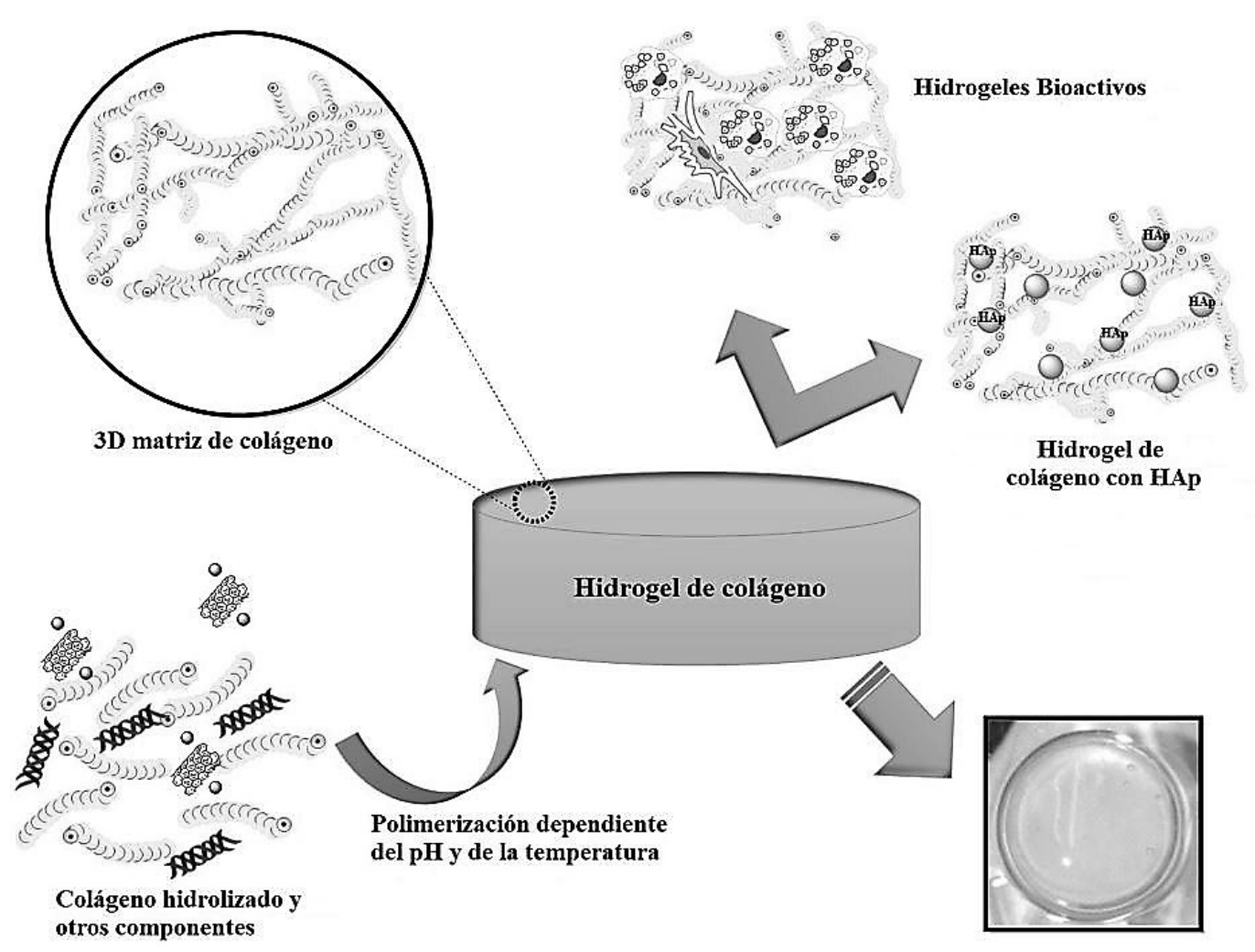

Figura 2. Hidrogel de colágeno con incorporación de HAp. Elaboración personal. 
Tabla III. Ejemplos de materiales incorporados a hidrogeles de colágeno.

\begin{tabular}{|c|c|c|}
\hline Material & Resultados/Propiedades & Referencias \\
\hline $\begin{array}{l}\text { Hidrogeles de nano-HAp/ } \\
\text { colágeno/alginato }\end{array}$ & $\begin{array}{l}\text { - Buenas propiedades biológicas y mecánicas; el hidrogel } \\
\text { compuesto exhibió un módulo de compresión de } \sim 230 \mathrm{MPa} \text { y } \\
\text { el módulo de tensión } \sim 33 \mathrm{MPa} \text {. } \\
\text { - Aumento en la citocompatibilidad, viabilidad celular y } \\
\text { proliferación acelerada. }\end{array}$ & $\begin{array}{c}\text { (Zheng, Jiang, Chen, Fan } \\
\text { \& Zhang, 2014) }\end{array}$ \\
\hline $\begin{array}{c}\text { Hidrogel de alginato/colágeno/ } \\
\text { HAp }\end{array}$ & $\begin{array}{l}\text { - Su módulo de compresión promedio alcanzó su punto máximo } \\
\text { a un valor de } \sim 0.0075 \mathrm{MPa} \text {, el cual puede ir aumentando con } \\
\text { el incremento de la concentración de calcio. } \\
\text { - Mayor osteoconductividad. }\end{array}$ & (Bendtsen \& Wei, 2015) \\
\hline $\begin{array}{l}\text { Hidrogeles compuestos de nano- } \\
\text { HAp/colágeno }\end{array}$ & - Alta proliferación celular y alto potencial de mineralización. & $\begin{array}{l}\text { (Hayrapetyan, Bongio, } \\
\text { Leeuwenburgh, Jansen \& } \\
\text { Van Den Beucken, 2016) }\end{array}$ \\
\hline $\begin{array}{l}\text { Andamios a base de quitosano/ } \\
\text { colágeno/ ácido hialurónico con } \\
\text { hidroxiapatita }\end{array}$ & $\begin{array}{l}\text { - Respuesta satisfactoria del tejido en los andamios implantados. } \\
\text { - Buena biocompatibilidad y crecimiento celular con la adición } \\
\text { de HAp. }\end{array}$ & (Kaczmarek et al., 2018) \\
\hline $\begin{array}{l}\text { Hidrogel nanocompuesto de } \\
\text { colágeno / hidroxiapatita y } \\
\text { quitosano }\end{array}$ & $\begin{array}{l}\text { - Buena citocompatibilidad. } \\
\text { - Mayor proliferación celular. }\end{array}$ & (Wang et al., 2009) \\
\hline $\begin{array}{c}\text { Hidrogel compuesto de } \\
\text { quitosano/nano-hidroxiapatita/ } \\
\text { colágeno }\end{array}$ & $\begin{array}{l}\text { - Forma un gel rápidamente estable a temperatura corporal por } \\
\text { lo que puede ser inyectado directamente. } \\
\text { - Muestra características en composición similares al hueso } \\
\text { natural. }\end{array}$ & $\begin{array}{c}\text { (Huang, Feng, Yu \& Li, } \\
\text { 2011a) }\end{array}$ \\
\hline $\begin{array}{l}\text { Hidrogel de colágeno/ quitosano/ } \\
\text { nano-hidroxiapatita }\end{array}$ & $\begin{array}{l}\text { - Actividades autoregenerativas y proliferativas hasta por } 20 \\
\text { días. Además de una buena citocompatibilidad. } \\
\text { - Mejoramiento de las propiedades mecánicas, presenta un mayor } \\
\text { módulo elástico a una presión ejercida de } \sim 0.001 \mathrm{MPa} \text {. }\end{array}$ & (Huang et al., 2011b) \\
\hline $\begin{array}{l}\text { Hidrogeles de colágeno/ } \\
\text { hidroxiapatita/alendronato }\end{array}$ & $\begin{array}{l}\text { - Propiedades mecánicas notablemente mejoradas (módulo de } \\
\text { elástico 0.038-0.187 MPa). } \\
\text { - Se favoreció la adhesión y crecimiento de las células } \\
\text { osteoblásticas. } \\
\text { - Excelente biocompatibilidad. }\end{array}$ & (Ma et al., 2016) \\
\hline Hidrogeles de colágeno/HAp & $\begin{array}{l}\text { - La incorporación de HAp mejoró la estabilidad mecánica del } \\
\text { hidrogel. Se aumentó la resistencia a la compresión. } \\
\text { - Buena biocompatibilidad y viabilidad celular. }\end{array}$ & $\begin{array}{l}\text { (Laydi, Rahouadj, } \\
\text { Cauchois, Stolz \& De } \\
\text { Isla, 2013) }\end{array}$ \\
\hline $\begin{array}{c}\text { Hidrogeles de colágeno/fibrina/ } \\
\text { HAp }\end{array}$ & $\begin{array}{l}\text { - Favorece la formación de la red endotelial. } \\
\text { - Se mejoran las propiedades mecánicas, mayor rigidez (Módulo } \\
\text { elástico } \sim 0.0003-0.00035 \mathrm{MPa} \text { ). }\end{array}$ & (Rao et al., 2014) \\
\hline $\begin{array}{l}\text { Hidrogel nanocompuesto } \\
\text { de colágeno/seda fibroína/ } \\
\text { hidroxiapatita }\end{array}$ & $\begin{array}{l}\text { - Distribución homogénea dentro de la matriz polimérica. } \\
\text { - Presenta un módulo de compresión de } \sim 200 \mathrm{MPa} \text {. } \\
\text { - Buena biocompatibilidad celular. }\end{array}$ & $\begin{array}{c}\text { (Chen, Hu, Ran, Shen \& } \\
\text { Tong, 2014) }\end{array}$ \\
\hline
\end{tabular}

y control del metabolismo de las células implicadas en los procesos de regeneración (Shen et al., 2011).

\section{Conclusiones}

La ingeniería de tejidos óseos representa un factor de suma importancia en la salud pública a nivel mundial, siendo el uso de hidrogeles de colágeno/HAp un facilitador para el reemplazo o la reparación del tejido dañado a consecuencia de lesiones o al desgaste debido a enfermedades relacionadas con el hueso, evitando técnicas quirúrgicas como autoinjerto o trasplantes. El desarrollo de biomateriales a base de hidrogeles de colágeno con la incorporación de HAp como materia inorgánica mejora las propiedades de biocompatibilidad, biodegradabilidad y mecánicas, además de que este tipo de hidrogeles compósitos 
presentan una mejor adhesión, proliferación, difusión y control del metabolismo celular implicado en la reparación ósea, siendo de vital importancia para su aplicación en ingeniería de tejidos. A la fecha, es necesario desarrollar nuevos métodos para la generación de estos materiales compósitos y evaluar su desempeño en la regeneración tisular ósea permitiendo mejorar la calidad de vida de la población que sufre este tipo de enfermedades.

\section{Agradecimientos}

Se agradece a la Universidad Autónoma de Coahuila, al Posgrado en Ciencia y Tecnología de Materiales CYTEMA y al Consejo Nacional de Ciencia y Tecnología (CONACYT) por el otorgamiento de la beca de doctorado No. 774498.

\section{REFERENCIAS}

Abou Neel, E. A., Cheema, U., Knowles, J. C., Brown, R. A. \& Nazhat, S. N. (2006). Use of multiple unconfined compression for control of collagen gel scaffold density and mechanical properties. Soft Matter, 2(11), 986-992. DOI:10.1039/b609784g.

Ahmed, E. M. (2015). Hydrogel: Preparation, characterization, and applications: A review. Journal of Advanced Research, 6(2), 105-121. DOI: 10.1016/j.jare.2013.07.006.

Al-Munajjed, A. A., Plunkett, N. A., Gleeson, J. P., Weber, T., Jungreuthmayer, C., Levingstone, T., Hammer, J. \& O'Brien, F. J. (2009). Development of a biomimetic collagenhydroxyapatite scaffold for bone tissue engineering using a SBF immersion technique. Journal of Biomedical Materials Research Part B: Applied Biomaterials, 90B(2), 584-591. DOI: $10.1002 / \mathrm{jbm} . b .31320$.

Ambekar, R. S. \& Kandasubramanian, B. (2019). Progress in the Advancement of Porous Biopolymer Scaffold: Tissue Engineering Application. Industrial \& Engineering Chemistry Research, 58(16), 6163-6194. DOI: 10.1021/ acs.iecr.8b05334.

Amini, A. R., Laurencin, C. T. \& Nukavarapu, S. P. (2012). Bone tissue engineering: recent advances and challenges. Critical Reviews in Biomedical Engineering, 40(5), 363-408.

Anseth, K. S., Bowman, C. N. \& Brannon-Peppas, L. (1996). Mechanical properties of hydrogels and their experimental determination. Biomaterials, 17(17), 1647-1657. DOI: 10.1016/0142-9612(96)87644-7.

Bahram, M., Mohseni, N., Moghtader, M. (2016). An introduction to hydrogels and some recent applications. Sutapa Biswas Majee, Intech Open (Ed.), Emerging Concepts in Analysis and Applications of Hydrogels, (pp. 9-22). DOI: 10.5772/64301.

Bai, X., Gao, M., Syed, S., Zhuang, J., Xu, X. \& Zhang, X.Q. (2018). Bioactive hydrogels for bone regeneration. Bioactive Materials, 3(4), 401-417. DOI: 10.1016/j. bioactmat.2018.05.006.

Bendtsen, S. T. \& Wei, M. (2015). Synthesis and characterization of a novel injectable alginate collagen hydroxyapatite hydrogel for bone tissue regeneration. Journal of Materials Chemistry B,3(15), 3081-3090. DOI: 10.1039/c5tb00072f.

Bharti,A., Singh, S., Meena, V. K. \& Goyal, N. (2016). Structural Characterization of Silver-Hydroxyapatite Nanocomposite: ABone Repair Biomaterial. Materials Today: Proceedings, 3(6), 2113-2120. DOI: 10.1016/j.matpr.2016.04.116.

Carlson, G. A., Dragoo, J. L., Samimi, B., Bruckner, D. A., Bernard, G. W., Hedrick, M. \& Benhaim, P. (2004). Bacteriostatic properties of biomatrices against common orthopaedic pathogens. Biochemical and Biophysical Research Communications, 321(2), 472-478. DOI: 10.1016/j.bbrc.2004.06.165.

Cen, L., Liu, W., Cui, L., Zhang, W. \& Cao, Y. (2008). Collagen Tissue Engineering: Development of Novel Biomaterials and Applications. Pediatric Research, 63(5), 492-496. DOI: 10.1203/PDR.0b013e31816c5bc3.

Claudio-Rizo, J. A., Rangel-Argote, M., Castellano, L. E., Delgado, J., Mata-Mata, J. L. \& Mendoza-Novelo, B. (2017). Influence of residual composition on the structure and properties of extracellular matrix derived hydrogels. Materials Science and Engineering: $C$, 79, 793-801. DOI: 10.1016/j.msec.2017.05.118.

Cosgriff-Hernandez,E. \& Mikos,A.G. (2008). New Biomaterials as Scaffolds for Tissue Engineering. Pharmaceutical Research, 25(10), 2345-2347. DOI: 10.1007/s11095-0089666-4.

Chai, Q., Jiao, Y. \& Yu, X. (2017). Hydrogels for Biomedical Applications: Their Characteristics and the Mechanisms behind Them. Gels (Basel, Switzerland), 3(1), 6. DOI: 10.3390/gels3010006.

Chen, L., Hu, J., Ran, J., Shen, X. \& Tong, H. (2014). Preparation and evaluation of collagen-silk fibroin/hydroxyapatite nanocomposites for bone tissue engineering. International Journal of Biological Macromolecules, 65, 1-7.DOI: 10.1016/j.ijbiomac.2014.01.003

Chen, L., Hu, J., Ran, J., Shen, X. \& Tong, H. (2016). Synthesis and cytocompatibility of collagen/hydroxyapatite nanocomposite scaffold for bone tissue engineering. Polymer Composites, 37(1), 81-90. DOI: 10.1002/pc.23157.

Chen, M. H., Wang, L. L., Chung, J. J., Kim, Y.-H., Atluri, P. \& Burdick, J. A. (2017). Methods to Assess Shear-Thinning Hydrogels for Application as Injectable Biomaterials. ACS Biomaterials Science \& Engineering, 3(12), 3146-3160. DOI: 10.1021 acsbiomaterials.7b00734.

De Witte, T. M., Fratila-Apachitei, L. E., Zadpoor,A.A. \& Peppas, N. A. (2018). Bone tissue engineering via growth factor delivery: from scaffolds to complex matrices. Regenerative Biomaterials, 5(4), 197-211. DOI: 10.1093/rb/rby013.

Dimitriou, R., Jones, E., McGonagle, D. \& Giannoudis, P. V. (2011). Bone regeneration: current concepts and future directions. BMC Medicine, 9(1), 66. DOI: 10.1186/17417015-9-66.

Drury, J. L. \& Mooney, D. J. (2003). Hydrogels for tissue engineering: scaffold design variables and applications. 
Biomaterials, 24(24), 4337-4351. DOI: 10.1016/s01429612(03)00340-5.

Fillingham, Y. \& Jacobs, J. (2016). Bone grafts and their substitutes. The Bone \& Joint Journal, 98-B, 6-9. DOI: 10.1302/0301-620x.98b.36350.

Froelich, K., Pueschel, R. C., Birner, M., Kindermann, J., Hackenberg, S., Kleinsasser, N. H., Hagen, R. \& Staudenmaier, R. (2010). Optimization of Fibrinogen Isolation for Manufacturing Autologous Fibrin Glue for Use as Scaffold in Tissue Engineering. Artificial Cells, Blood Substitutes, and Biotechnology, 38(3), 143-149. DOI: $10.3109 / 10731191003680748$.

Gallo, N., Nasser, H., Salvatore, L., Natali, M. L., Campa, L., Mahmoud, M., Capobianco, L., Sannino, A. \& Madaghiele, M. (2019). Hyaluronic acid for advanced therapies: Promises and challenges. European Polymer Journal, 117, 134-147. DOI: 10.1016/j.eurpolymj.2019.05.007.

Gardin, C., Ferroni, L., Favero, L., Stellini, E., Stomaci, D., Sivolella, S., Bressan, E. \& Zavan, B. (2012). Nanostructured biomaterials for tissue engineered bone tissue reconstruction. International Journal of Molecular Sciences, 13(1), 737-757. DOI: 10.3390/ijms13010737.

Gea, S., Surga, M., Rahayu, S., Marpongahtun, Hutapea, Y. A. \& Piliang, A. F. (2018). The analysis of thermal and mechanical properties of biocomposite policaprolactone/ cellulose nanofiber from oil palm empty fruit bunches. AIP Conference Proceedings, 2049(1), 020063. DOI: 10.1063/1.5082468.

George, A., Shah, P. A. \& Shrivastav, P. S. (2019). Natural biodegradable polymers-basednano-formulations for drug delivery:A review.InternationalJournalofPharmaceutics, 561, 244-264. DOI: 10.1016/j.ijpharm.2019.03.011.

Giannoudis, P. V., Dinopoulos, H. \& Tsiridis, E. (2005). Bone substitutes: An update. Injury, 36(3), S20-S27. DOI: 10.1016/j.injury.2005.07.029.

Hayrapetyan, A., Bongio, M., Leeuwenburgh, S. C., Jansen, J.A. \& Van Den Beucken, J. J. (2016). Effect of Nano-HA/ Collagen Composite Hydrogels on Osteogenic Behavior of Mesenchymal Stromal Cells. Stem Cell Reviews and Reports, 12(3), 352-364. DOI: 10.1007/s12015-0169644-x.

Huang, G. \& Chen, J. (2019). Preparation and applications of hyaluronic acid and its derivatives. International Journal of Biological Macromolecules, 125, 478-484.DOI: 10.1016/j. ijbiomac.2018.12.074.

Huang, Z., Feng, Q., Yu, B. \& Li, S. (2011a). Biomimetic properties of an injectable chitosan/nano-hydroxyapatite/ collagen composite. Materials Science and Engineering: C, 31(3), 683-687. DOI: 10.1016/j.msec.2010.12.014.

Huang, Z., Yu, B., Feng, Q., Li, S., Chen, Y. \& Luo, L. (2011b). In situ-forming chitosan/nano-hydroxyapatite/collagen gel for the delivery of bone marrow mesenchymal stem cells. Carbohydrate Polymers, 85(1), 261-267. DOI:10.1016/j. carbpol.2011.02.029.
Hutmacher, D. W., Schantz, J. T., Lam, C. X., Tan, K. C., \& Lim, T. C. (2007). State of the art and future directions of scaffoldbased bone engineering from a biomaterials perspective. Journal of Tissue Engineering and Regenerative Medicine, 1(4), 245-260. DOI: 10.1002/term.24.

Imre, B. \& Pukánszky, B. (2013). Compatibilization in biobased and biodegradable polymer blends. European Polymer Journal, 49(6), 1215-1233. DOI: 10.1016/j. eurpolymj.2013.01.019.

Jansen, K. A., Licup, A. J., Sharma, A., Rens, R., MacKintosh, F. C. \& Koenderink, G. H. (2018). The Role of Network Architecture in Collagen Mechanics. Biophysical Journal, 114(11), 2665-2678. DOI: 10.1016/j.bpj.2018.04.043.

Joon, B. P. (1984). Biomaterials science and engineering. Springer, Boston, MA. ISBN 978-1-4612-9710-9. DOI: 10.1007/978-1-4613-2769-1 1.

Kaczmarek, B., Sionkowska, A., Gołyńska, M., Polkowska, I., Szponder, T., Nehrbass, D. \& Osyczka, A. M. (2018). In vivo study on scaffolds based on chitosan, collagen, and hyaluronic acid with hydroxyapatite. International Journal of Biological Macromolecules, 118, 938-944. DOI: 10.1016/j.ijbiomac.2018.06.175.

Kalita, S. J., Bhardwaj,A.\& Bhatt, H.A.(2007). Nanocrystalline calcium phosphate ceramics in biomedical engineering. Materials Science and Engineering: C, 27(3), 441-449. DOI: 10.1016/j.msec.2006.05.018.

Kaviani, A., Zebarjad, S. M., Javadpour, S., Ayatollahi, M. \& Bazargan-Lari, R. (2019). Fabrication and characterization oflow-cost freeze-gelated chitosan/collagen/hydroxyapatite hydrogel nanocomposite scaffold. International Journal of Polymer Analysis and Characterization, 24(3), 191-203. DOI: 10.1080/1023666x.2018.1562477.

Khan, F. \& Ahmad, S. R. (2013). Polysaccharides and Their Derivatives for Versatile Tissue Engineering Application. Macromolecular Bioscience, 13(4), 395-421. DOI: 10.1002/ mabi.201200409.

Kuriakose, T.A., Kalkura, S. N., Palanichamy, M., Arivuoli, D., Dierks, K., Bocelli, G. \& Betzel, C. (2004). Synthesis of stoichiometric nano crystalline hydroxyapatite by ethanolbased sol-gel technique at low temperature. Journal of Crystal Growth, 263(1), 517-523. DOI:10.1016/j. jcrysgro.2003.11.057.

Labet, M. \& Thielemans, W. (2009). Synthesis of polycaprolactone: a review. Chemical Society Reviews, 38(12), 3484-3504. DOI: 10.1039/b820162p.

Lammi, M. J., Piltti, J., Prittinen, J. \& Qu, C. (2018). Challenges in Fabrication of Tissue-Engineered Cartilage with Correct Cellular Colonization and Extracellular Matrix Assembly. International Journal of Molecular Sciences, 19(9), 2700. DOI: $10.3390 / \mathrm{ijms} 19092700$.

Laurencin, C. T., Ambrosio, A. M. A., Borden, M. D. \& Cooper, J.A. (1999). Tissue Engineering: Orthopedic Applications. Annual Review of Biomedical Engineering, 1(1), 19-46. DOI: 10.1146/annurev.bioeng.1.1.19. 
Laydi, F., Rahouadj, R., Cauchois, G., Stolz, J. F. \& De Isla, N. (2013). Hydroxyapatite incorporated into collagen gels for mesenchymal stem cell culture. Bio-Medical Materiales andEngineering, 23,311-315.DOI: 10.3233/BME-130755.

Lee, B., Jo, S., Kim, S., Cho, M., Park, S., Youn, J., Ji, J. D. \& Kim, T. (2018). Poly- $\gamma$-glutamic acid suppresses osteoclastogenesis in human osteoclast precursors and prevents joint damage in a collagen-induced murine arthritis model. Immunology Letters, 203, 80-86. DOI: 10.1016/j. imlet.2018.09.004.

Li, Z., Su, Y., Xie, B., Wang, H., Wen, T., He, C., Shen, H., Wu, D. \& Wang, D. (2013). A tough hydrogel-hydroxyapatite bone-like composite fabricated in situ by the electrophoresis approach. Journal of Materials Chemistry B, 1(12), 17551764. DOI: 10.1039/c3tb00246b.

Lickorish, D., Ramshaw, J.A. M., Werkmeister, J.A., Glattauer, V. \& Howlett, C. R. (2004). Collagen-hydroxyapatite composite prepared by biomimetic process. Journal of Biomedical Materials Research Part A, 68A(1), 19-27. DOI: 10.1002/jbm.a.20031.

Lin, K. \& Chang, J. (2015). 1 - Structure and properties of hydroxyapatite for biomedical applications. In M. Mucalo (Ed.), Hydroxyapatite (HAp) for Biomedical Applications, (pp. 3-19). Woodhead Publishing.

Ma, X., He, Z., Han, F., Zhong, Z., Chen, L. \& Li, B. (2016). Preparation of collagen/hydroxyapatite/alendronate hybrid hydrogels as potential scaffolds for bone regeneration. Colloids and Surfaces B: Biointerfaces, 143, 81-87. DOI: 10.1016/j.colsurfb.2016.03.025.

Martin-Piedra, A. \& Martin-Piedra, L. (2019). Matrices para ingeniería del tejido óseo. Actualidad Médica, 104(806), 36-45. DOI:10.15568/am.2019.806.re01.

Murugan, R. \& Ramakrishna, S. (2005). Development of nanocomposites for bone grafting. Composites Science and Technology, 65(15), 2385-2406.DOI: 10.1016/j. compscitech.2005.07.022.

Nasiri-Tabrizi, B., Fahami, A. \& Ebrahimi-Kahrizsangi, R. (2013). Effect of milling parameters on the formation of nanocrystalline hydroxyapatite using different raw materials. Ceramics International, 39(5), 5751-5763. DOI: 10.1016/j.ceramint.2012.12.093.

O'Brien, F. J. (2011). Biomaterials \& scaffolds for tissue engineering. Materials Today, 14(3), 88-95. DOI: 10.1016/ S1369-7021(11)70058-X.

Oftadeh, R., Perez-Viloria, M., Villa-Camacho, J. C., Vaziri, A. \& Nazarian, A. (2015). Biomechanics and mechanobiology of trabecular bone: a review. Journal of Biomechanical Engineering, 137(1), 0108021-01080215. DOI: 10.1115/1.4029176.

Orive, G., Hernández, R. M., Garcón, A., Igartúa, M. \& Pedráz M., J. L. (2003). Ingeniería Tisular: Retos y Realidades. Vitae, 10(2), 46-51. https://www.redalyc.org/articulo. oa? id=169817981005.

Parenteau-Bareil, R., Gauvin, R. \& Berthod, F. (2010). Collagen-
Based Biomaterials for Tissue Engineering Applications. Materials, 3(3), 1863-1887. DOI: 10.3390/ma3031863.

Parikh, S. (2002). Bone graft substitutes: past, present, future. Journal of Postgraduate Medicine, 48(2), 142-148.http:// www.jpgmonline.com/text.asp?2002/48/2/142/123.

Peng, F., Yu, X. \& Wei, M. (2011). In vitro cell performance on hydroxyapatite particles/poly(l-lactic acid) nanofibrous scaffolds with an excellent particle along nanofiber orientation. Acta Biomaterialia, 7(6), 2585-2592. DOI: 10.1016/j.actbio.2011.02.021.

Porter, J. R., Ruckh, T. T. \& Popat, K. C. (2009). Bone tissue engineering: A review in bone biomimetics and drug delivery strategies. Biotechnology Progress, 25(6), 15391560. DOI: 10.1002/btpr.246.

Ramli, R. A., Adnan, R., Bakar, M. A \& Masudi, S. M. (2011). Synthesis and characterization of pure nanoporous hydroxyapatite. Journal of Physical Science, 22(1), 25-37.

Rao, R. R., Ceccarelli, J., Vigen, M. L., Gudur, M., Singh, R., Deng, C. X., Putnam, A. J. \& Stegemann, J. P. (2014). Effects of hydroxyapatite on endothelial network formation in collagen/fibrin composite hydrogels in vitro and in vivo. Acta Biomaterialia, 10(7), 3091-3097. DOI: 10.1016/j. actbio.2014.03.010.

Rincón, M., Rodríguez, A., Londoño, M. E. \& Echavarría, A. (2007). Fabricación y caracterización de una matriz tridimensional de hidroxiapatita macroporosa para aplicación en ingeniería de tejidos óseos. Revista EIA, 87-95. http://www.scielo.org.co/scielo.php?script=sci_ar ttext\&pid=S179412372007000100008\&lng=en\&tlng=es.

Roberts, T. T. \& Rosenbaum, A. J. (2012). Bone grafts, bone substitutes and orthobiologics. Organogenesis, 8(4), 114124. DOI: 10.4161 /org. 23306.

Sachlos, E., Reis, N., Ainsley, C., Derby, B. \& Czernuszka, J. T. (2003). Novel collagen scaffolds with predefined internal morphology made by solid freeform fabrication. Biomaterials, 24(8), 1487-1497.DOI: 10.1016/S01429612(02)00528-8.

Sadat-Shojai, M., Khorasani, M.-T., Dinpanah-Khoshdargi, E \& Jamshidi, A. (2013). Synthesis methods for nanosized hydroxyapatite with diverse structures. Acta Biomaterialia, 9(8), 7591-7621. DOI: 10.1016/j.actbio.2013.04.012.

Salgado, A. J., Coutinho, O. P. \& Reis, R. L. (2004). Bone Tissue Engineering: State of the Art and Future Trends. Macromolecular Bioscience, 4(8), 743-765. DOI: 10.1002/ mabi.200400026.

Sequeda, L. G., Díaz, J. M., Gutiérrez, S. J., Perdomo, S. J. \& Gómez, O. L. (2012). Obtención de hidroxiapatita sintética por tres métodos diferentes y su caracterización para ser utilizada como sustituto óseo. Revista Colombiana de Ciencias Químico-Farmacéuticas, 41(1), 50-66.

Shen, X., Chen, L., Cai, X., Tong, T., Tong, H. \& Hu, J. (2011). A novel method for the fabrication of homogeneous hydroxyapatite/collagen nanocomposite and nanocomposite scaffold with hierarchical porosity. Journal of Materials 
Science: Materialsin Medicine, 22(2), 299-305. DOI: 10.1007/s10856-010-4199-x.

Sherman, V. R., Yang, W. \& Meyers, M.A. (2015). The materials science of collagen. Journal of the Mechanical Behavior of Biomedical Materials, 52, 22-50. DOI: 10.1016/j. jmbbm.2015.05.023.

Shi, L., Yang, N., Zhang, H., Chen, L., Tao, L., Wei, Y., Liu, H. \& Luo, Y. (2015). A novel poly $(\gamma$-glutamic acid $) /$ silk-sericin hydrogel for wound dressing: Synthesis, characterization and biological evaluation. Materials Science and Engineering: C, 48, 533-540. DOI: 10.1016/j. msec.2014.12.047.

Shuk, P., Suchanek, W. L., Hao, T., Gulliver, E., Riman, R. E., Senna, M., TenHuisen, K. S. \& Janas, V. F. (2001). Mechanochemical-hydrothermal preparation of crystalline hydroxyapatite powders at room temperature. Journal of Materials Research, 16(5), 1231-1234. DOI: 10.1557/ jmr.2001.0170.

Silva, C. C., Pinheiro, A. G., Miranda, M. A. R., Góes, J. C. \& Sombra, A. S. B. (2003). Structural properties of hydroxyapatite obtained by mechanosynthesis. Solid State Sciences, 5(4), 553-558. DOI: 10.1016/S12932558(03)00035-9.

Sommerfeldt, D. \& Rubin, C. (2001). Biology of bone and how it orchestrates the form and function of the skeleton. European Spine Journal, 10(2), S86-S95. DOI: 10.1007/ s005860100283.

Stock, S. R. (2015). The Mineral-Collagen Interface in Bone. Calcified Tissue International, 97(3), 262-280. DOI: 10.1007/s00223-015-9984-6.

Suchanek, W. \& Yoshimura, M. (1998). Processing and properties of hydroxyapatite-based biomaterials for use as hard tissue replacement implants. Journal of Materials Research, 13(1), 94-117. DOI: 10.1557/jmr.1998.0015.

Szcześ, A., Hołysz, L. \& Chibowski, E. (2017). Synthesis of hydroxyapatite for biomedical applications. Advances in Colloid and Interface Science, 249, 321-330. DOI: 10.1016/j.cis.2017.04.007.

Takallu, S., Mirzaei, E., Azadi, A., Karimizade, A. \& Tavakol, S. (2019). Plate-shape carbonated hydroxyapatite/collagen nanocomposite hydrogel via in situ mineralization of hydroxyapatite concurrent with gelation of collagen at $\mathrm{pH}$ $=7.4$ and $37^{\circ} \mathrm{C}$. Journal of Biomedical Materials Research Part B: Applied Biomaterials, 107(6), 1920-1929. DOI: 10.1002/jbm.b.34284.

Tan, H. \& Marra, K. G. (2010). Injectable, Biodegradable Hydrogels for Tissue Engineering Applications. Materials, 3(3), 1746-1767. DOI: 10.3390/ma3031746.

Tolaimate, A., Desbrieres, J., Rhazi, M. \& Alagui, A. (2003). Contribution to the preparation of chitins and chitosans with controlled physico-chemical properties. Polymer, 44(26), 7939-7952.DOI: 10.1016/j.polymer.2003.10.025.

Turnbull, G., Clarke, J., Picard, F., Riches, P., Jia, L., Han, F., Li, B. \& Shu, W. (2018). 3D bioactive composite scaffolds for bone tissue engineering. Bioactive Materials, 3(3), 278-
314. DOI: 10.1016/j.bioactmat.2017.10.001.

Vallet-Regí, M. (2010). Evolution of bioceramics within the field of biomaterials. Comptes Rendus Chimie, 13(1), 174-185. DOI: 10.1016/j.crci.2009.03.004.

Wahl D. A. \& Czernuszka, J. (2006). Collagen-hydroxyapatite composites for hard tissue repair. European Cells and Materials, 11, 43-56. DOI: 10.22203/eCM.v011a06.

Wang, W. \& Yeung, K. (2017). Bone grafts and biomaterials substitutes for bone defect repair: A review. Bioactive Materials, 2(4), 224-247. DOI: 10.1016/j. bioactmat.2017.05.007.

Wang, X., Wang, X., Tan, Y., Zhang, B., Gu, Z. \& Li, X. (2009). Synthesis and evaluation of collagen-chitosanhydroxyapatite nanocomposites for bone grafting. Journal of Biomedical Materials Research Part A, 89A(4), 10791087. DOI: 10.1002/jbm.a.32087.

Wang, Y., Von Euw, S., Fernandes, F. M., Cassaignon, S., Selmane, M., Laurent, G., Pehau-Arnaudet, G., Coelho, C., Bonhomme-Coury, L., Giraud-Guille, M.-M., Babonneau, F.,Azaïs, T. \& Nassif, N.(2013). Water-mediated structuring of bone apatite. Nature Materials, 12, 1144. DOI: 10.1038/ nmat3787.

Xu, J., Tan, X., Chen, L., Li, X. \& Xie, F. (2019). Starch/ microcrystalline cellulose hybrid gels as gastric-floating drug delivery systems. Carbohydrate Polymers, 215, 151159. DOI: 10.1016/j.carbpol.2019.03.078.

Yahia, S., Khalil, I. A. \& El-Sherbiny, I. M. (2019). SandwichLike Nanofibrous Scaffolds for Bone Tissue Regeneration. ACS Applied Materials \& Interfaces, 11(32), 28610-28620. DOI: 10.1021 /acsami.9b06359.

Zhang, D., Wu, X., Chen, J. \& Lin, K. (2018). The development of collagen based composite scaffolds for bone regeneration. Bioactive Materials, 3(1), 129-138. DOI: 10.1016/j. bioactmat.2017.08.004

Zhang, L.J., Feng, X.S., Liu, H.G., Qian, D.J., Zhang, L., Yu, X.L. \& Cui, F.Z. (2004). Hydroxyapatite/collagen composite materials formation in simulated body fluid environment. Materials Letters, 58(5), 719-722. DOI: 10.1016/j.matlet.2003.07.009.

Zhao, H., Ma, L., Gao, C. \& Shen, J. (2008). Fabrication and properties of mineralized collagen-chitosan/hydroxyapatite scaffolds. Polymers for Advanced Technologies, 19(11), 1590-1596. DOI:10.1002/pat.1174.

Zheng, L., Jiang, X., Chen, X., Fan, H. \& Zhang, X. (2014). Evaluation of novelinsitusynthesizednano-hydroxyapatite/ collagen/alginate hydrogels for osteochondral tissue engineering. Biomedical Materials, 9(6), 065004. DOI: 10.1088/1748-6041/9/6/065004.

Zhou, H. \& Lee, J. (2011). Nanoscale hydroxyapatite particles for bone tissue engineering. Acta Biomaterialia, 7(7), 27692781. DOI: 10.1016/j.actbio.2011.03.019.

Zivanovic, S., Davis, R. H. \& Golden, D. A. (2015). 8 - Chitosan as an antimicrobial in food products. In T. M. Taylor (Ed.), Handbook of Natural Antimicrobials for Food Safety and Quality (pp. 153-181). Oxford: Woodhead Publishing. 